\title{
Enhanced migration and adhesion of peripheral blood neutrophils from SAPHO patients revealed by RNA-Seq
}

\author{
Yuxiu Sun ${ }^{1 \dagger}$, Chen $\mathrm{Li}^{2+}$, Mengmeng Zhu', Shen Zhang ${ }^{1}$, Yihan Cao ${ }^{3}$, Qiao Yang ${ }^{4}$, Pengfei Zhao ${ }^{1}$, \\ Guangrui Huang ${ }^{1 *}$ and Anlong $\mathrm{Xu}^{1,5^{*}}$
}

\begin{abstract}
Background: SAPHO syndrome is a rare disease characterized by inflammatory lesions on skin and bones. Diversified manifestation and inadequate understanding of etiology has limited its diagnosis and treatment. The co-occurrence of other immune-mediated diseases strongly suggests an involvement of autoimmunity in SAPHO syndrome. However, the role of the largest population of circulating immune cells, neutrophils, is still not well explored. In this study, we performed RNA sequencing to profile the mRNA expression of neutrophils purified from peripheral blood of SAPHO patients to identify key genes associated with SAPHO syndrome, trying to find new functional molecules or biomarkers for this rare disease.
\end{abstract}

Results: A total of 442 differentially expressed genes were identified ( $p<0.05$, fold change $>2$ ), in which 294 genes were upregulated and 148 genes were downregulated. Five differentially expressed genes of interest were verified by quantitative Real-Time Polymerase Chain Reaction (qRT-PCR), among which S100A12 was upregulated and positively related to high-sensitivity C-reactive protein (hsCRP), while the downregulated gene MYADM was positively related to osteocalcin. Kyoto Encyclopedia of Genes and Genomes (KEGG) analysis showed that differentially expressed genes were enriched in "systemic lupus erythematosus" and "ECM-receptor interaction". Gene ontology (GO) enrichment showed that differentially expressed genes may participate in biological processes such as "cell migration" and "cell adhesion".

Conclusions: In conclusion, this study provides a first insight into transcriptome characteristics of SAPHO syndrome, indicating an over-active neutrophil recruitment in patients and possibly suggesting molecular candidates for further study on diagnosis and pathology of this disease.

Keywords: SAPHO syndrome, RNA-Seq, Neutrophil, Cell adhesion, Cell migration

\section{Introduction}

SAPHO syndrome is a relatively rare disease known under the acronym of SAPHO (Synovitis, Acne, Pustulosis, Hyperostosis and Osteitis), which was first named by French rheumatologists in 1987 [1]. It is characterized by a wide range of dermatological and osteoarticular lesions, involvement of characteristic target sites (such as anterior chest wall, axial skeleton and pelvic bones [2]), and a clinical course of recurrently relapses and remissions without

\footnotetext{
* Correspondence: hgr@bucm.edu.cn; xuanlong@bucm.edu.cn

${ }^{\dagger}$ Yuxiu Sun and Chen Li contributed equally to this work.

${ }^{1}$ School of Life Sciences, Beijing University of Chinese Medicine, Beijing, China

Full list of author information is available at the end of the article
}

specific histopathologic features [3]. Lacking of validated molecular diagnostic criteria, the diagnosis of SAPHO syndrome is challenging and mainly based on clinical and radiological findings [4]. The fact that dermatological and osteoarticular lesions may occur many years apart or even not both present on the same patient always leads to delayed diagnosis, with patients suffering ongoing pain and skin manifestations [5].

Although the pathogenesis of SAPHO syndrome largely remains unknown, the significantly exhibited overlap and co-occurrence of manifestations of other immunemediated diseases such as ankylosing spondylitis, psoriasis vulgaris and inflammatory bowel disease (IBD) [6] strongly

(c) The Author(s). 2019 Open Access This article is distributed under the terms of the Creative Commons Attribution 4.0 International License (http://creativecommons.org/licenses/by/4.0/), which permits unrestricted use, distribution, and reproduction in any medium, provided you give appropriate credit to the original author(s) and the source, provide a link to the Creative Commons license, and indicate if changes were made. The Creative Commons Public Domain Dedication waiver (http://creativecommons.org/publicdomain/zero/1.0/) applies to the data made available in this article, unless otherwise stated. 
suggests an inflammatory response, possibly involving autoimmunity [7]. Several previous studies have explored the role of immune cells in the development and prognosis of SAPHO syndrome. For instance, natural killer cells were found to be significantly reduced [8], while peripheral Th17 cells were reported to be increased in patients with SAPHO syndrome [9]. As for the largest population of innate immune cells, neutrophils, existing evidence has been limited. Although enhanced neutrophil infiltration was observed in PSTPIP2-deficient mice which presented a SAPHO syndrome-like phenotype [10], no significant increase in serum neutrophils in patients had been found. Neutrophils purified from patients with SAPHO syndrome were reported to have an abnormal internal oxidant production following stimulation with phorbol myristate acetate (PMA) [11], but no signs of aberrant intercellular oxidant production was shown [12]. Until recently, there has not been further investigation on the role of neutrophils in SAPHO syndrome. Meanwhile, it has been well understood that neutrophils play multifaceted roles in autoimmune diseases [13] and neutrophil-derived chemokines contribute to the pathogenesis of non-infectious diseases [14]. Thus, our study focused on the differentially expressed mRNAs in peripheral neutrophils from patients with SAPHO syndrome, trying to descript how the most abundant leucocytes, as well as one of the earliest immune cells to arrive at the inflammatory sites in the human body participate in SAPHO syndrome.

Genome-wide RNA sequencing (RNA-Seq) and bioinformatics analysis have been widely applied in clinical and basic research to identify key mRNAs that regulate autoimmune diseases $[15,16]$. However, the molecular function and clinical value of mRNAs profile in SAPHO syndrome are still not explored. In this study, we performed RNASeq and bioinformatics analysis to identify key mRNAs associated with SAPHO syndrome, trying to find new functional molecules or biomarkers for this rare disease.

\section{Results}

\section{Clinical features of included individuals}

Six SAPHO patients and six healthy volunteers were recruited between March 2017 and June 2018. The historical disease status and clinical manifestation of each patient are shown in Table 1. All six patients suffered from bone lesions, with five on anterior chest wall, six on axial skeleton, two on peripheral joints and one on mandible. Skin involvement was observed in five patients, among which palmoplantar pustulosis is most commonly presented (5/6). Patient \#1 was the only one without cutaneous lesions, whose osteoarticular manifestations were similar to the others except for an additional involvement of mandible. As shown in Table 2, there was no significant difference in age or gender between SAPHO group and Control group. Although the levels of peripheral white blood cells and neutrophils are slightly higher in patients with SAPHO syndrome, no statistical significance was found ( $p=0.101$ and $p=0.117$, respectively). The mean visual analogue scale (VAS) of recruited patients is 4.50 , indicating a middle-to-severe pain of infected bones. Compared with healthy controls, high-sensitivity $\mathrm{C}$ reactive protein (hsCRP) is slightly higher in patients. Besides, three of the patients exhibited faster erythrocyte sedimentation rate (ESR), while two had a lower level of serum osteocalcin, although the average levels of ESR and osteocalcin are within the normal range.

\section{Overview of RNA-Seq}

To systematically analyze mRNAs related to SAPHO syndrome, approximately 1.15 billion reads for 12 samples from 6 patients and 6 healthy controls were sequenced using RNA-Seq (Additional file 1: Table S1). Experimental and computational scheme for the RNASeq process is shown on Fig. 1a. Firstly, the total RNA from neutrophils was isolated and reverse-transcribed into cDNA library. RNA sequencing was then performed on Illumina HiSeq 2000 Platform. Clean data was mapped to GRCh38 with HISAT2 [17] and then assembled into transcripts. After data homogenization and quality control, the expression level of each transcript was calculated by fragments per kilobase of exon per million fragments mapped (FPKM). The boxplot of FPKM values of all transcripts indicated similar whole transcriptome expression in each sample (Fig. 1b) and principal component analysis (PCA) revealed a relatively

Table 1 Disease status and clinical manifestation of each patient

\begin{tabular}{llll}
\hline Patients \# & $\begin{array}{l}\text { Disease duration } \\
\text { (months) }\end{array}$ & Clinical manifestation & \\
\cline { 3 - 4 } & 10 & Skin & Bone (affected part) \\
2 & 108 & Palmoplantar pustulosis & anterior chest wall, axial skeleton, mandible \\
3 & 6 & Palmoplantar pustulosis & anterior chest wall, axial skeleton \\
4 & 7 & Palmoplantar pustulosis & axial skeleton, peripheral joint \\
5 & 6 & Palmoplantar pustulosis & anterior chest wall, axial skeleton, peripheral joint \\
6 & 24 & Palmoplantar pustulosis, psoriasis vulgaris & anterior chest wall, axial skeleton \\
\hline
\end{tabular}


Table 2 Demographics and clinical parameters of included individuals

\begin{tabular}{|c|c|c|c|c|}
\hline & Control & SAPHO & Reference range & $p$ value \\
\hline Gender & female & female & & 1 \\
\hline Age & $25.33 \pm 1.75$ & $33.16 \pm 10.26$ & & 0.053 \\
\hline White Blood Cell $\left({ }^{*} 10^{9} / \mathrm{L}\right)$ & $5.63 \pm 0.95$ & $7.27 \pm 2.02$ & $4.0-10.0$ & 0.101 \\
\hline Neutrophil $\left({ }^{*} 10^{9} / \mathrm{L}\right)$ & $3.00 \pm 0.81$ & $4.24 \pm 1.57$ & $1.8-6.3$ & 0.117 \\
\hline $\operatorname{VAS}(1-10)$ & - & $4.50 \pm 2.59$ & $0-10$ & - \\
\hline hsCRP (mg/L) & - & $3.03 \pm 3.63$ & $0-3$ & - \\
\hline ESR $(\mathrm{mm} / \mathrm{h})$ & - & $15 \pm 8.67$ & $0-20$ & - \\
\hline Osteocalcin (ng/L) & - & $3.73 \pm 1.83$ & $1.8-8.4$ & - \\
\hline
\end{tabular}

\section{A}

RNA isolation from peripheral neutrophils

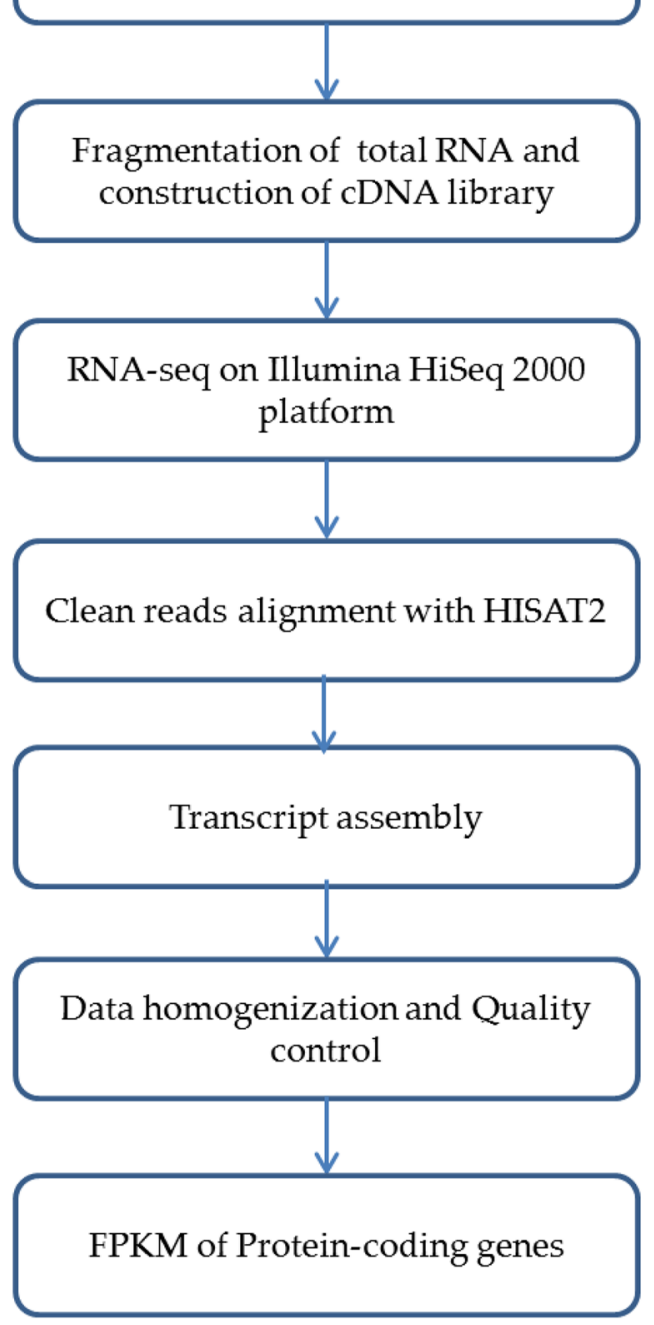

B

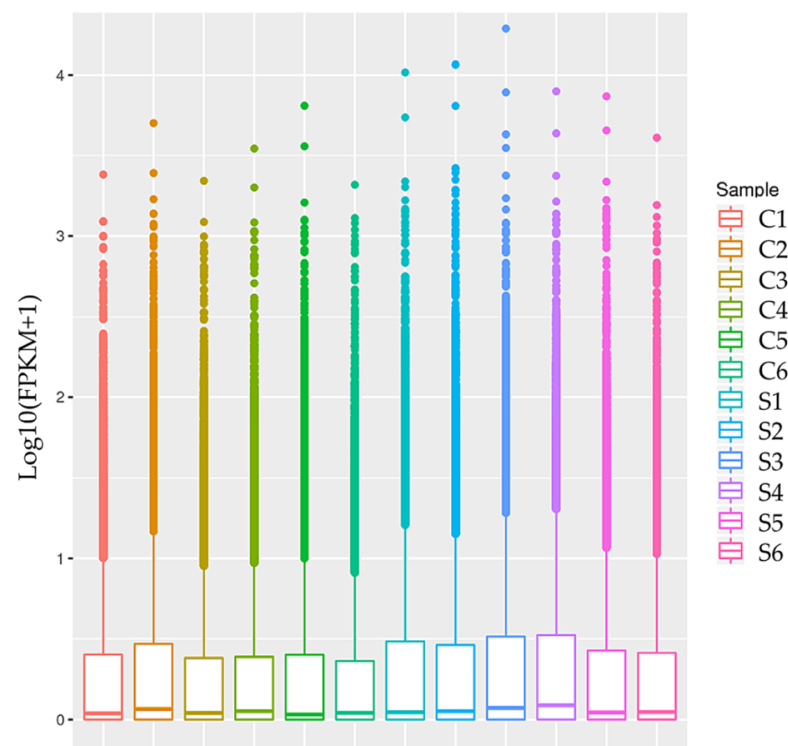

C

$\begin{array}{lllllllllllllllll}\mathrm{C} 1 & \mathrm{C} 2 & \mathrm{C} 3 & \mathrm{C} 4 & \mathrm{C} 5 & \mathrm{C} 6 & \mathrm{~S} 1 & \mathrm{~S} 2 & \mathrm{~S} 3 & \mathrm{~S} 4 & \mathrm{~S} 5 & \mathrm{~S} 6\end{array}$

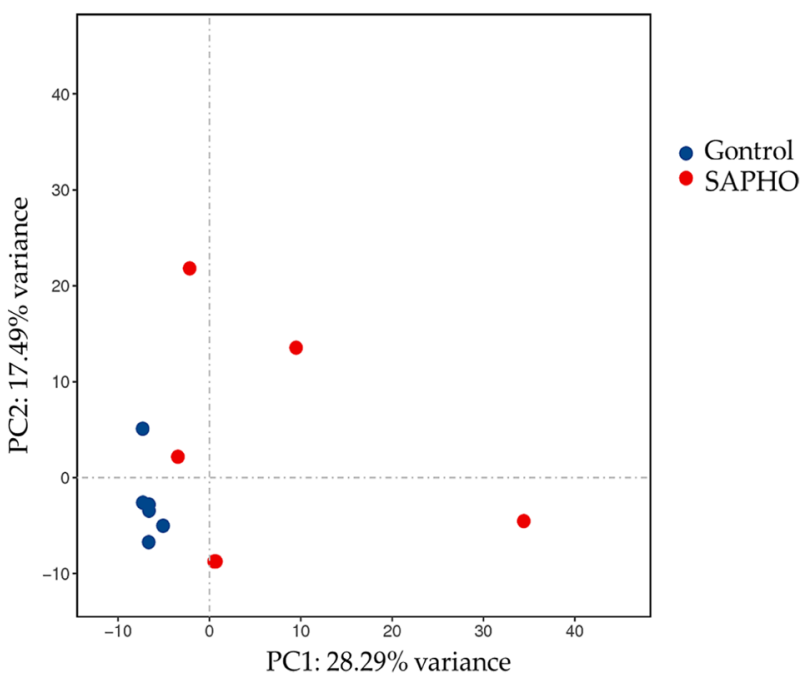

Fig. 1 Overview of RNA-Seq. a RNA-Seq procedure. $\mathbf{b}$ Boxplot for FPKM values of all transcripts in each sample. c PCA map of all samples in Control and SAPHO group 
clear distinction between samples in the two groups along the first principal component of $28.29 \%$ variance (Fig. 1c).

\section{Differentially expressed genes in SAPHO patients}

The differentially expressed mRNAs were identified between SAPHO group and Control group using " $P<0.05$, fold change $>2$ " as a cutoff (Fig. 2a). The Hierarchical Clustering analysis was conducted to illustrate the distinguishable expression pattern between the two groups (Fig. 2b). A total of 442 differentially expressed genes were identified, among which 294 were up-regulated and 148 down-regulated (Additional file 2: Table S2). As reported before, tumor necrosis factor- $\alpha$ (TNF- $\alpha$ ) signaling plays important role in the pathogenesis of SAPHO syndrom [18], while IL6, IL17 and IL18 were reported to be elevated in the sera of SAPHO patients [19-21]. We found that TNF- $\alpha$ receptor encoding genes TNFRSF1B, IL6 receptor subunit IL6ST, IL17 receptor gene IL17RA and IL18 receptor genes IL18R1 were up-regulated in peripheral blood neutrophils of SAPHO patients (Fig. 2c). These results indicated that peripheral neutrophils of SAPHO patients were in a more active status than those of the healthy controls.

\section{Validation of differentially expressed genes and correlation with disease parameters}

Five differentially expressed genes of interest were selected for the validation by qRT-PCR (Fig. 3a) in a larger population (12 patients and 12 gender-age matched healthy controls). Among them, S100 Calcium Binding Protein A12 (S100A12) has been detected in salivary proteome profile
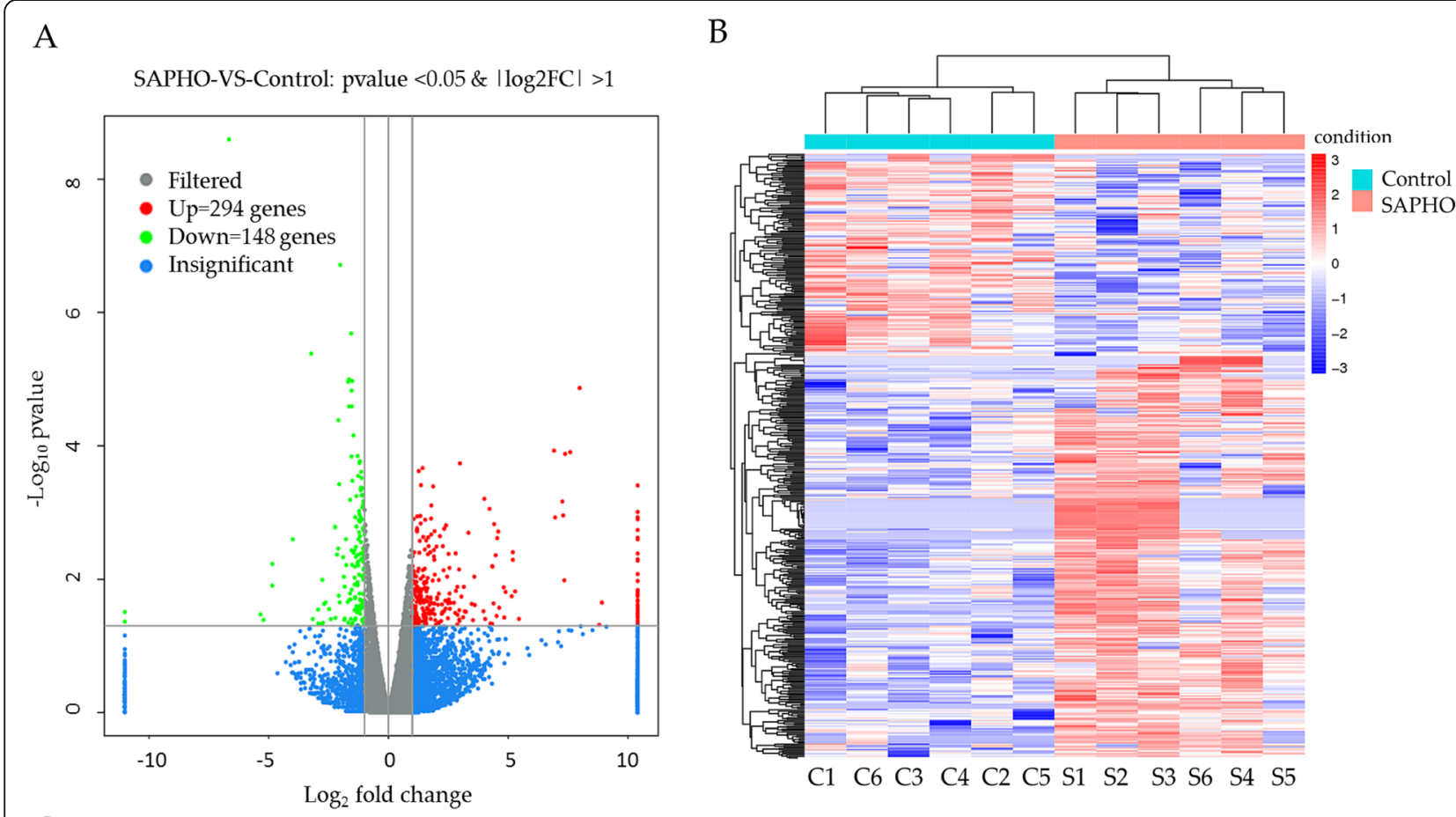

C
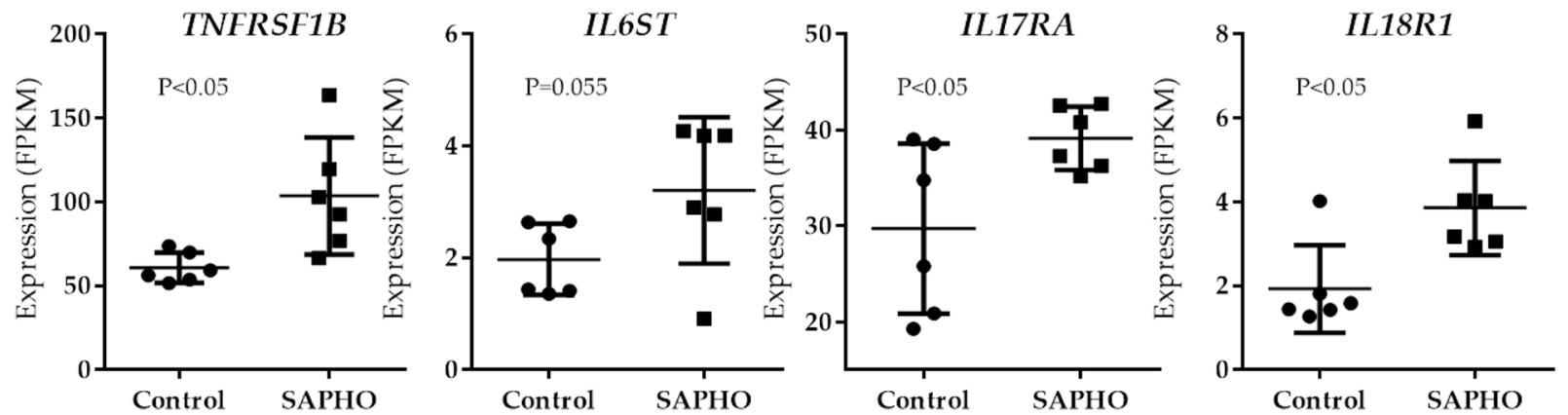

Fig. 2 Differentially expressed genes in patients with SAPHO syndrome. a Differentially expressed genes were identified using "fold change > 2.00 and $P$ value $<0.05^{\prime \prime}$ as a cutoff. b Hierarchical Clustering analysis of differentially expressed genes in SAPHO patients showed closer relationship within groups. c Gene expression (FPKM) of TNFRSF1B, IL6ST, IL17RA and IL18R1, p value was calculated by Student t-test 


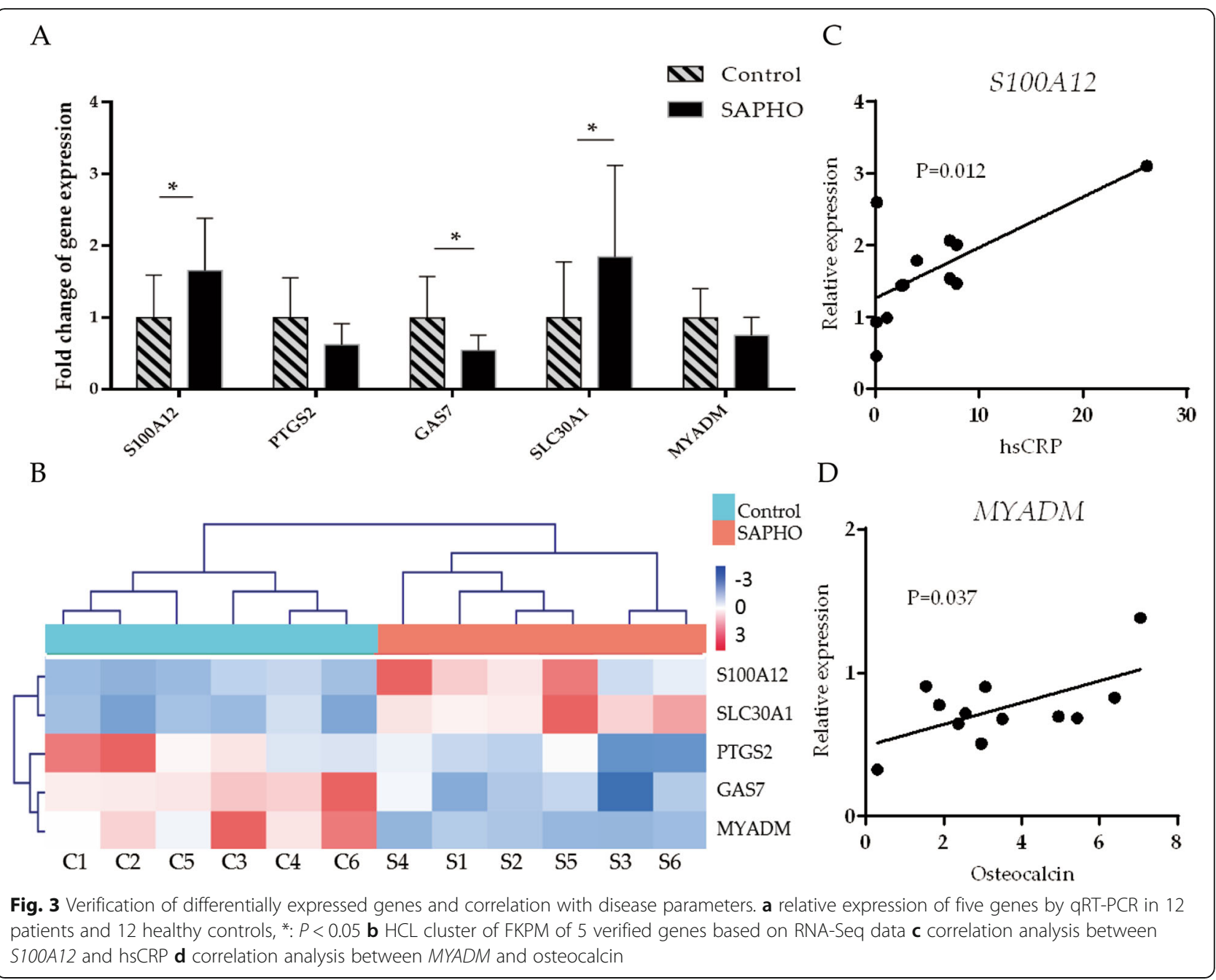

of SAPHO syndrome [22]. Prostaglandin-endoperoxide synthase 2 (PTGS2 or COX-2) was down-regulated in chronic inflammation [23], whose inhibitors are considered as a potential risk factor for bone healing [24]. In the meanwhile, growth-arrest-specific 7 gene (GAS7) is required for osteoblast differentiation [25], which may play a role in bone manifestations in SAPHO syndrome. Besides, a feedback loop between inflammation and $\mathrm{Zn}$ uptake has been observed in rheumatoid arthritis [26], while $\mathrm{Zn}$ transporters, also known as SLC30 families, are related to different skin disorders [27] as well as systemic inflammatory response syndrome [28]. Besides, myeloid-associated differentiation marker (MYADM) drew our attention because of its important role in cell spreading and migration [29], which is consistent with our findings in GO term enrichment analysis. The expression levels of S100A12 and SLC30A1 were up regulated while those of PTGS2, GAS7 and $M Y A D M$ were down-regulated in SAPHO patients, which was in accordance with the RNA-Seq data (Fig. 3b). However, not all the differences in gene expression were statistically significant, and the verification in a larger population is still necessary. To further investigate the biological significance of differentially expressed genes, their correlation with clinical parameters was analyzed. Interestingly, a positive correlation was observed between S100A12 and peripheral hsCRP (Fig. 3c), indicating a role in inflammation. Besides, the down-regulated gene $M Y A D M$ was positively correlated to osteocalcin (Fig. 3d), which is essential for bone metabolism.

\section{KEGG pathway enrichment of differentially expressed genes}

Kyoto Encyclopedia of Genes and Genomes (KEGG) analysis showed that differentially expressed genes were mainly enriched in pathways about autoimmunity, infection, cancer and metabolism (Fig. 4). Among them, the involvement of system lupus erythematosus (SLE) drew our attention. Similar to SAPHO syndrome, SLE is an autoimmune disease with diverse presentations ranging from rash and arthritis to anemia, serositis, nephritis, seizures, etc., while incidence of both diseases is higher in women aged 15-50 [30]. Anti-nucleosome antibodies 


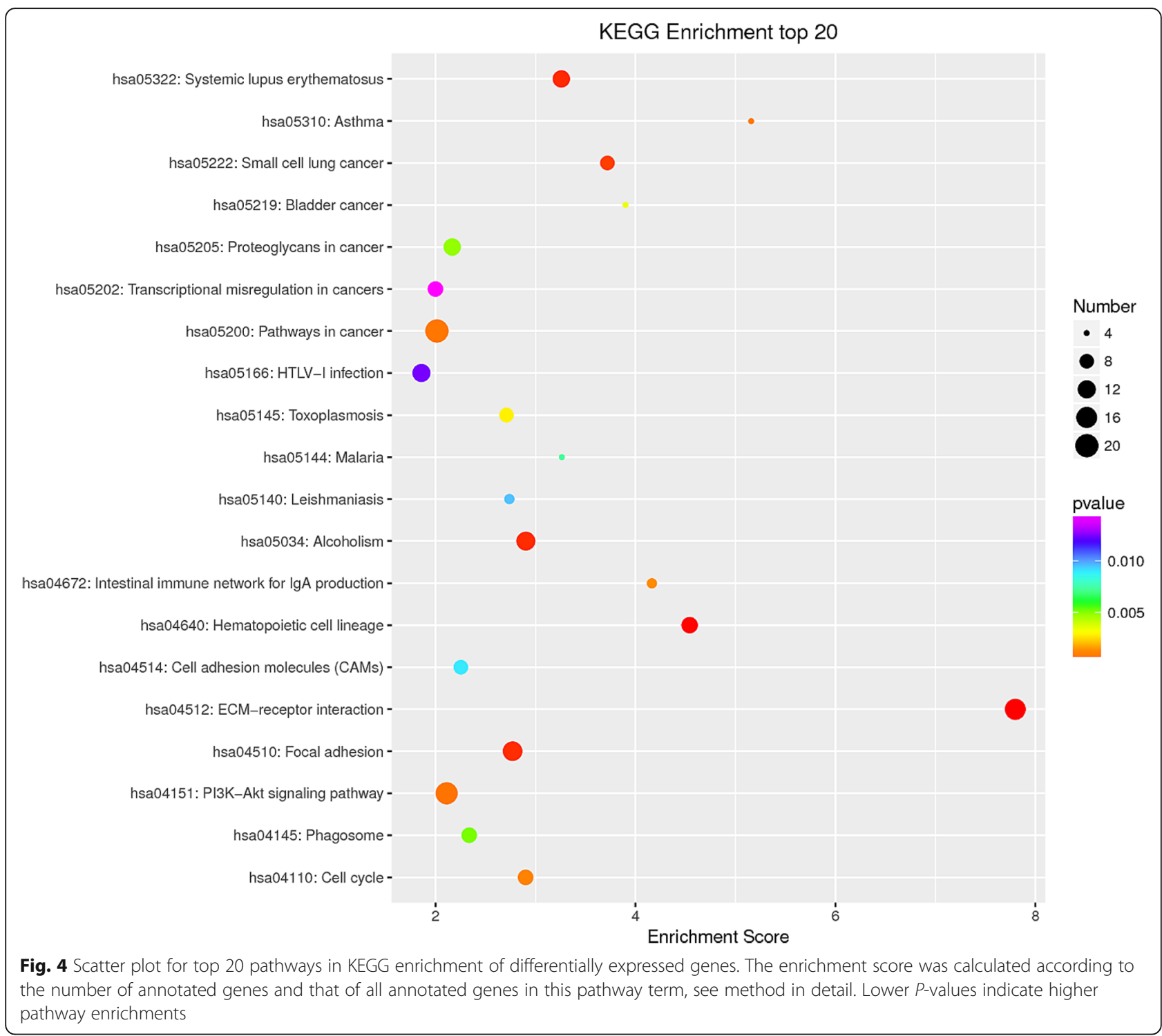

as well as anti-dsDNA antibodies are present and essential in the diagnosis of patients with SLE [31], while mRNAs encoding histone-derived peptides such as $H 2 A$, $H 2 B, H 3$ and $H 4$ were up-regulated in SAPHO group. The activation of SLE pathway in patients may implicate an immune-mediated etiology in SAPHO syndrome. Besides, extracellular matrix (ECM)-receptor interaction is also among the top 20 significant pathways, which was commonly associated with poor prognosis of cancer [32], and recent study also revealed its association with osteoarthritis [33]. Genes enriched in each pathway are shown in Additional file 3: Table S3.

\section{Functional prediction of differentially expressed genes in GO database}

The top 10 significantly enriched terms of biological process, cellular component and molecular function in
Gene Ontology (GO) analysis are shown in Fig. 5. In terms of biological process, differentially expressed genes were predicted to be involved in GO terms such as cell adhesion, platelet degranulation and peripheral nervous system development. The most significantly indicated terms were extracellar matrix in cell component and IgE binding in molecular function. Interestingly, differentially expressed genes were enriched in cell migration (14 genes enriched) and cell adhesion (28 genes enriched), which were both important for neutrophils recruitment in response to inflammation. Genes enriched in each GO term are shown in Additional file 4: Table S4.

\section{PPI network among genes enriched in cell adhesion and migration}

To gain insight into how neutrophil adhesion and migration are regulated in patients with SAPHO syndrome, the 


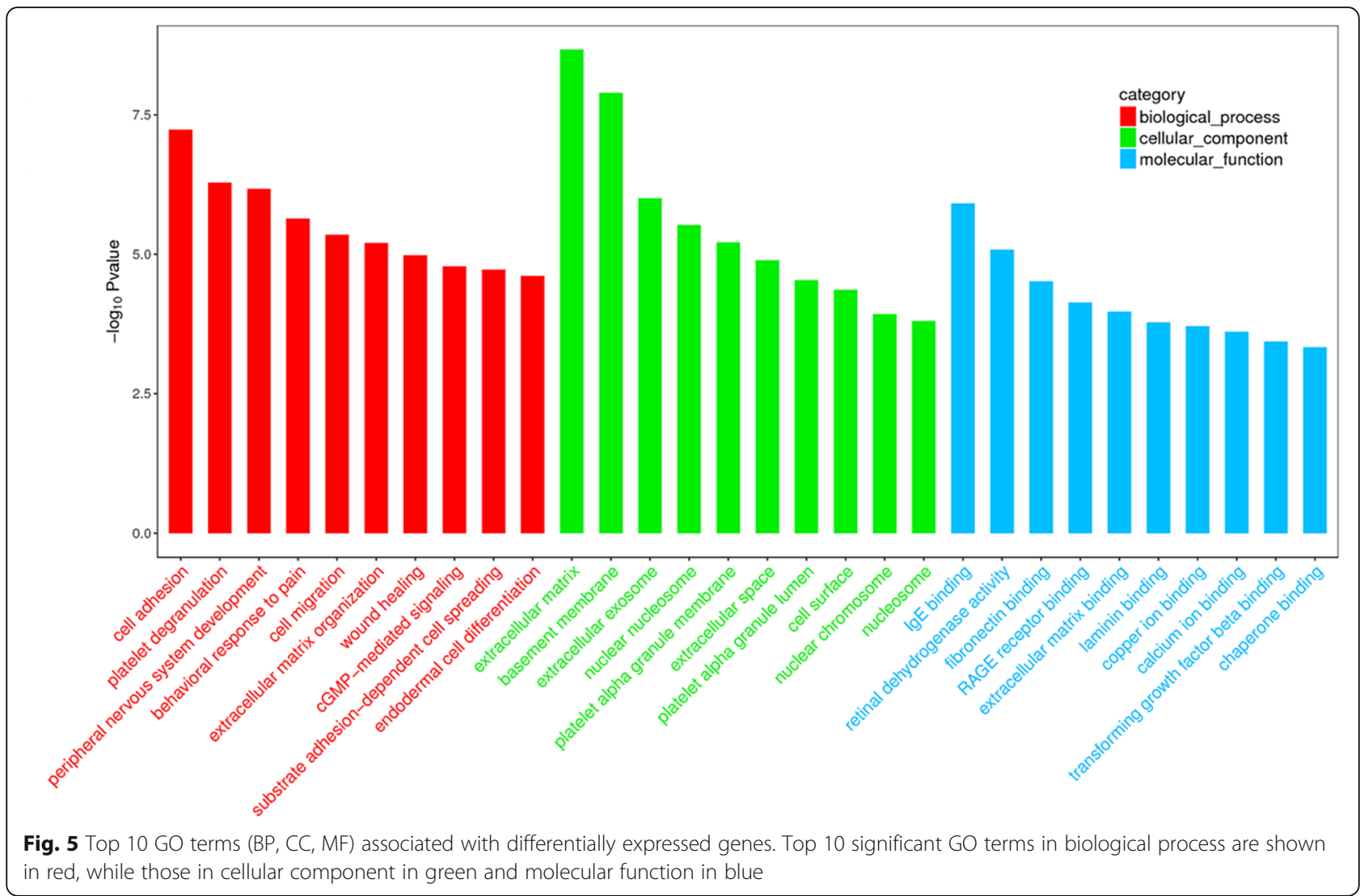

network of protein-protein interaction (PPI) among genes enriched in cell adhesion and cell migration was constructed based on STRING database (Fig. 6a). The up-regulation of hub genes in the network indicated an over-activated cell adhesion and migration in patients with SAPHO syndrome (Fig. 6b). Among them were components of laminin (LAMA4, LAMB1 and LAMC1) and melanoma cell adhesion molecule $(M C A M)$, which functions as a receptor for LAMA4 [34], promoting cell adhesion and slow-rolling of neutrophils [35]. Up-regulation of tenascin-C (TNC), an endogenous ECM molecule required in neutrophil recruitment [36], was also observed in SAPHO group.

\section{Enhanced neutrophil recruitment in SAPHO patients}

As previously described, the recruitment of neutrophils consists of priming, rolling, arrest, adhesion, crawling, and migration [37]. Specifically, resting neutrophils in peripheral blood are primed by stimuli such as cytokines and interaction with activated endothelial cells, which leads to active transcription and expression of receptors or other molecules [38], among which the P-selectin glycoprotein ligand 1 (PSGL-1) expressed by neutrophils binds in high on-and-off-rate to P-selectins on the endothelial cells and mediates the rolling step [39]. Afterwards, the endothelial cells present chemokines which activate lymphocyte function-associated antigen-1 (LFA-1) and macrophage-1 antigen (Mac-1) on neutrophils, promoting neutrophil arrest [40]. Neutrophils then crawl along the surface of the endothelium to a suitable extravasation site (Fig. 7a). Besides, as we further scanned the differentially expressed genes, several additional genes were found to play important roles in neutrophil recruitment, including the up-regulated gene ITGB2, which is component of LFA-1 and Mac-1. Another increased gene, C-C chemokine receptor-like 2 (CCRL2), has also been proved to be crucial in neutrophil recruitment at sites of inflammation [41]. Other genes such as CD302, a C-type lectin receptor involved in cell adhesion and migration [42] and CD151, known to complex with integrins and other transmembrane 4 superfamily proteins [43], were also upregulated in SAPHO syndrome (Fig. 7b).

\section{Discussion}

SAPHO syndrome is a chronic and recurrently disease characterized by cutaneous and osteoarticular inflammation. Both diagnosis and treatment for this rare disease have been limited by its heterogeneous manifestation and elusive etiology. Circulating neutrophils are very sensitive to inflammatory signals and are the first pioneers recruited to the inflammatory site [13]. Besides, enhanced neutrophil infiltration was observed in mice with a SAPHO syndrome-like phenotype [10]. In this 


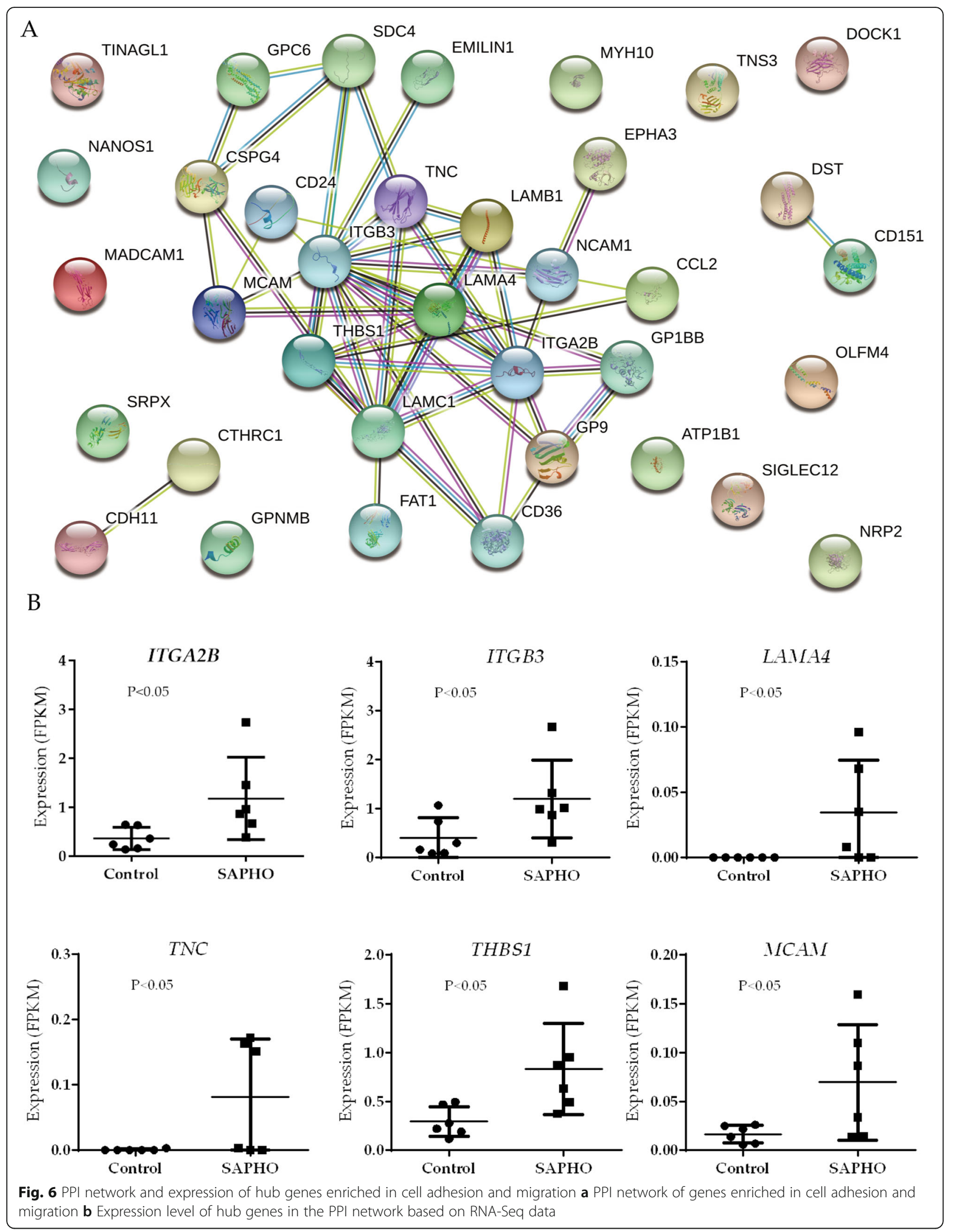




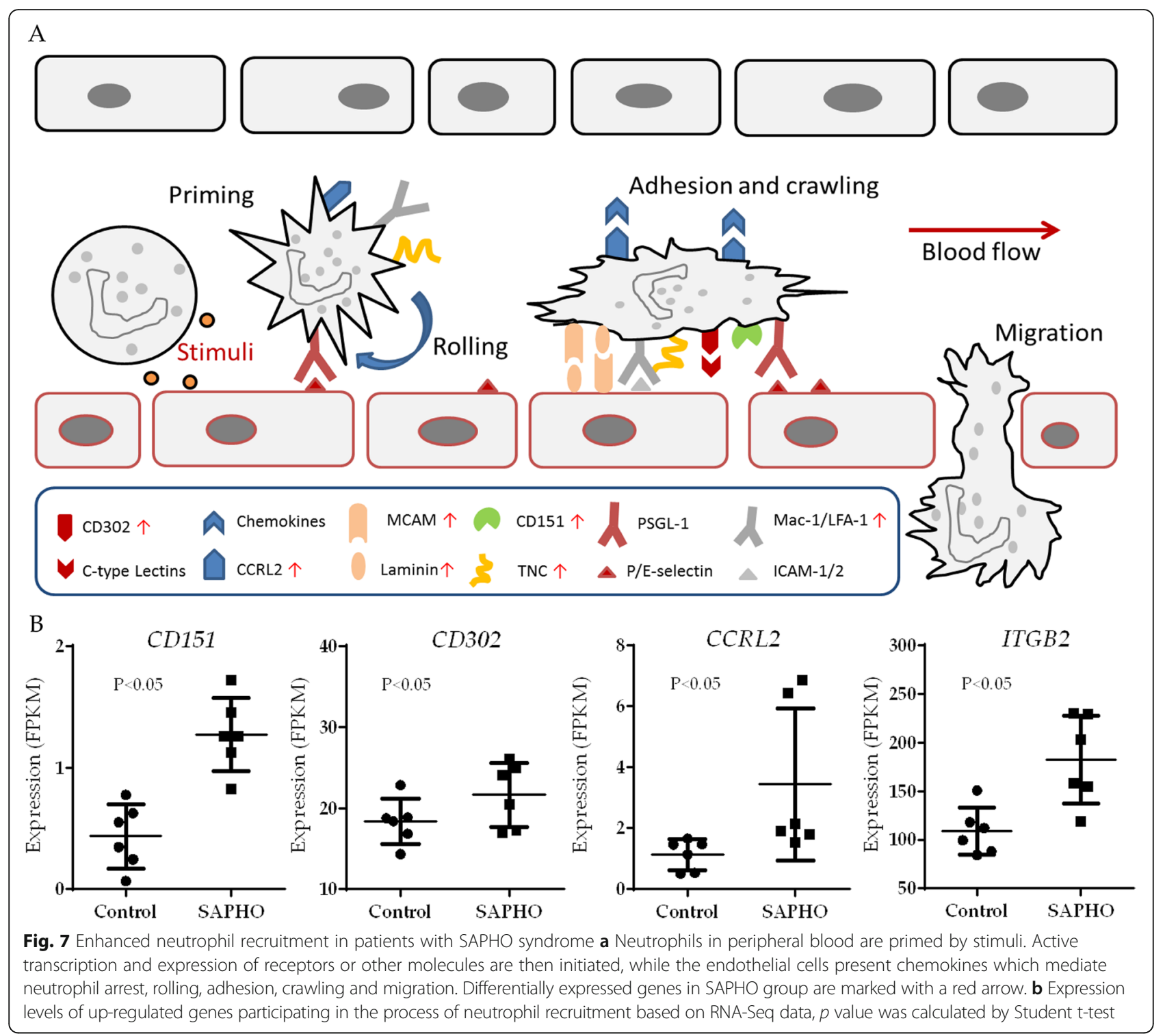

study, RNA-Seq was performed to profile the mRNA expression level of neutrophils purified from peripheral blood of SAPHO patients. A total of 442 differentially expressed genes were identified (294 upregulated and 148 downregulated). It has been reported that TNF- $\alpha$ signaling pathway plays an important role in the pathogenesis of SAPHO [18], while IL6, IL17 and IL18 were elevated in the serum of SAPHO patients [19-21]. Genes encoding the receptors for TNF $\alpha$, IL6, IL17 and IL18 were up-regulated in peripheral blood neutrophils of SAPHO patients. KEGG analysis showed that differentially expressed genes were enriched in "systemic lupus erythematosus" and "ECM-receptor interaction". GO enrichment suggested that differentially expressed genes may function by participating in biological processes such as "cell migration" and "cell adhesion". These results indicated that the peripheral blood neutrophils in SAPHO patients might have enhanced activation and migration potential.

Five differentially expressed genes of interest were validated by qRT-PCR, among which S100A12 was observed to be elevated in the saliva of SAPHO patients [22] and serum S100A12 is related to juvenile idiopathic arthritisassociated uveitis [44]. Besides, S100A12 secreted by neutrophils is a regulator of reactive oxygen species and potassium efflux [45]. PTGS2 was down-regulated in SAPHO patients, which was also previously reported in chronic inflammation [23]. As PTGS2 inhibitors are considered to be a potential risk factor for bone healing [24], we suspect that the down-regulation of PTGS2 in SAPHO syndrome may contribute to the osteoarticular lesions in patients. The results of qRT-PCR were approximately in consistence with RNA-Seq data. S100A12 was elevated in neutrophils from patients with SAPHO 
syndrome in our study, and the expression level is positively related to serum hsCRP. Meanwhile, the downregulated gene $M Y A D M$ was positively related to serum osteocalcin in SAPHO patients. Osteocalcin is mainly expressed by osteoclast and plays a key role in bone endocrine functions [46]. The down-regulation of MYADM may contribute to bone metabolism and delayed bone recovery in SAPHO syndrome. Thus, these genes may serve as potential markers of disease activity in clinical treatment instruction. However, the mechanism how these genes participated in the etiology and development of SAPHO syndrome requires further investigation.

To gain insight into the characterization of biological functions of differentially expressed genes between SAPHO group and the control group, GO term enrichment and KEGG pathway analysis were also performed. Eleven of the differentially expressed genes were enriched in SLE pathway, indicating that an autoimmune dysfunction may be involved in SAPHO syndrome. Pathologically, the histone-derived peptides $\mathrm{H}_{2} \mathrm{~B}_{10-33}, \mathrm{H} 4_{16-39}, \mathrm{H} 4_{71-94}$, $\mathrm{H} 3_{91-105}, \mathrm{H} 2 \mathrm{~A}_{34-48}$, and $\mathrm{H}_{49-63}$ stimulated $\mathrm{T}$ cells from patients with lupus to produce cytokines, which can help $B$ cells respond to antigenic epitopes of nucleosomes. Meanwhile, the role of neutrophils in SLE has been indicated as the proteins derived from neutrophil extracellular traps (NETs) may serve as autoantigens [47] and that infiltrating neutrophils in skin and kidney contribute to the pathology of SLE by undergoing NETosis and releasing nucleic acids and proteins [48]. We found that mRNAs of $H 2 A, H 2 B, H 3$ and $H 4$ are also up-regulated in neutrophils of SAPHO patients, which indicated that neutrophils of SAPHO patients may be prone to NETosis.

The role of neutrophil migration in inflammation has drawn increased interest in the past decade and recent evidences suggested that complex neutrophil behavior of forward and reverse migration contribute to resolution of inflammation [49]. In this study, 28 of the differentially expressed genes were enriched in the GO term of cell adhesion and 14 in cell migration. Based on the STRING database, we identified the PPI network of these genes and several hub regulators in the biological process. The up-regulation of hub genes in the network indicated an over-activation of cell adhesion and cell migration in SAPHO syndrome. Specifically, resting neutrophils are primed by inflammation and active transcription of genes such as TNC, CCRL2 and ITGB2 (CD18) are initiated. The up-regulated ITGB2 is component of LFA-1 and Mac-1, which interact with ICAM on endothelial cells, promoting neutrophil arrest [40]. Components of laminin (LAMA4, LAMB1 and LAMC1) and $M C A M$ also help cell adhesion and slow-rolling of neutrophils [35], the up-regulation of whom indicated excessive neutrophil activation and migration in SAPHO syndrome. Other up-regulated genes such as CD302 and
CD151 were also reported to play important roles in cell adhesion and migration [42,43]. Thus, we speculated that over-active neutrophil adhesion and migration may contribute to the pathology of SAPHO syndrome, which is in consistence with the excessive neutrophil infiltration on multiple organs in SAPHO patients.

\section{Conclusion}

In summary, our research provided the first insight into peripheral blood neutrophil mRNA profile for SAPHO patients, offering potential biomarkers for disease diagnosis and prognosis. We found that enhanced migration and adhesion of peripheral blood neutrophils may play important roles in the pathogenesis of SAPHO syndrome, which indicated that inhibition of neutrophil migration and adhesion might be a candidate therapeutic strategy for SAPHO syndrome.

\section{Materials and methods \\ Subjects and samples}

This study was approved by the Institutional Review Board of Peking Union Medical College Hospital (protocol number: ZS-994). We recruited 12 patients with SAPHO syndrome (informing consent obtained) between March 2017 and June 2018. As controls, we included 12 healthy volunteers in the same gender and age range. $20 \mathrm{ml}$ peripheral blood sample was collected from each participant and neutrophils were purified with Polymorphprep ${ }^{\text {to }}$ by gradient centrifugation (neutrophil purities $>90 \%$ ).

\section{Sequencing and analysis of RNA expression}

The total RNA was isolated from neutrophils using Trizol reagent (Invitrogen Life Technologies, Carlsbad, CA, USA). Qualified RNA (rRNA removed) was reverse transcribed into cDNA after fragmentation. RNA sequencing was constructed on Illumina HiSeq 2000 Platform. Clean data was mapped to the reference database GRCh38 with HISAT2 [17], while Bowtie2 [50] and eXpress [51] were used to assemble the transcripts. The expression level of RNA was calculated by fragments per kilobase of exon per million fragments mapped (FPKM). Differentially expressed mRNAs between Control group and SAPHO group were determined by DEseq [52] with " $p<0.05$, fold change $>2$ " as the threshold for significance.

\section{Quantitative real-time PCR}

Relative expression levels of selected genes were determined by quantitative real-time PCR (qRT-PCR). $200-500 \mathrm{ng}$ of total RNA was reverse transcribed to cDNA using a qPCR RT Master Mix with gDNA Remover Kit (TOYOBO). QRT-PCR was performed on Biorad iQ5 machine using a SYBR Green Realtime PCR Master MixPremix (TOYOBO) according to the manufacturer's instruction. All experiments were conducted in 
three technical replicates. The relative expression level of each target gene was normalized to the expression of $\beta$-actin with the $2^{-\Delta \Delta C t}$ method. Primers are shown in Additional file 5: Table S5.

\section{GO term enrichment and KEGG pathway analysis}

Differentially expressed genes were selected for functional annotation. Terms of biological process, cellular component and molecular function were analyzed according to Gene Ontology (GO) database (http://amigo. geneontology.org/amigo/landing) and functional pathway analysis were based on Kyoto Encyclopedia of Genes and Genomes (KEGG) database (http://www.kegg.jp/). Hypergeometric distribution test was used to determine the significance of differentially expressed mRNA enrichment in each GO term or KEGG pathway. The enrichment score was evaluated as follows:

$$
\text { Enrichment score }={ }^{\frac{m}{n} /} \frac{M}{N}
$$

Here, $\mathrm{N}$ is the number of GO-annotated or KEGGannotated mRNAs in all genes, while $\mathrm{n}$ is the number of GO-annotated or KEGG-annotated mRNAs in differentially expressed genes in N. M is the number of mRNAs annotated to a particular GO term or KEGG pathway in all genes while $\mathrm{m}$ is the number of genes annotated to a particular GO term or KEGG pathway in differently expressed genes.

\section{Statistical analysis}

Continuous variables were presented as means \pm standard deviation. Student's t-test was performed using SPSS version 20.0 software for comparisons between groups. The correlation of gene expression and clinical parameters was performed using Pearson's correlation coefficient. $P$ value $<0.05$ was considered statistically significant.

\section{Additional files}

Additional file 1: Table S1. RNA-Seq data summary. Amount of reads aquired by RNA-Seq and quality control data summary of each sample. (XLSX $10 \mathrm{~kb}$ )

Additional file 2: Table S2. Differentially expressed genes. List of differentially expressed genes in SAPHO syndrome, related $P$ value, fold change, and up/down regulation of genes were shown on Additional file 1: Table S1. (XLSX 37 kb)

Additional file 3: Table 3. Genes enriched in top 20 KEGG pathways. $P$ value, enrichment score, and list of genes enriched in each particular pathway of the top $20 \mathrm{KEGG}$ pathways involved in SAPHO syndrome. (XLSX $11 \mathrm{~kb}$ )

Additional file 4: Table S4. Genes enriched in top $10 \mathrm{GO}$ terms of BP,CC,MF. P value, enrichment score, and list of genes enriched in each $\mathrm{GO}$ term of the top $10 \mathrm{GO}$ terms of $\mathrm{BP}, \mathrm{CC}, \mathrm{MF}$ involved in SAPHO syndrome. (XLSX $14 \mathrm{~kb}$ )

Additional file 5: Table 5. Primers of differentially expressed genes. Sequence of foward and reverse primers used in the qRT-PCR verification of differentially expressed genes. (XLSX $9 \mathrm{~kb}$ )

\section{Abbreviations}

CCRL2: C-C chemokine receptor-like 2; ECM: Extracellular metrix;

ESR: Erythrocyte sedimentation rate; FPKM: Fragments per kilobase of exon per million fragments mapped; GAS7: Growth-arrest-specific 7; GO: Gene Ontology; hsCRP: High sensitivity C-Reactive protein; IBD: Inflammatory bowel disease; ITGB2: Integrin beta 2; KEGG: Kyoto Encyclopaedia of Genes \& Genomes; LFA-1: Lymphocyte function-associated antigen-1; Mac-1: Macrophage1 antigen; MCAM: Melanoma cell adhesion molecule; MYADM: Myeloid-associated differentiation marker; NETs: Neutrophil extracellular traps; PCA: Principal component analysis; PMA: Phorbol myristate acetate; PPI: Protein-protein interaction; PSGL-1: P-selectin glycoprotein ligand 1; PTGS2: Prostaglandinendoperoxide synthase 2; RNA-Seq: RNA sequencing; S100A12: S100 Calcium Binding Protein A12; SAPHO: Synovitis, Acne, Pustulosis, Hyperostosis and Osteitis; SLE: System lupus erythematosus; TNC: Tenascin-C; TNF-a: Tumor necrosis factor-a; VAS: Visual analogue scale

\section{Acknowledgements}

Not applicable.

\section{Authors' contributions}

Conceptualization, GH and AX; Data curation, MZ and YC; Formal analysis, YS, $\mathrm{CL}$ and $\mathrm{SZ}$; Funding acquisition, $\mathrm{AX}, \mathrm{GH}$ and $\mathrm{CL}$; Investigation, $\mathrm{YS}$ and $\mathrm{QY}$; Methodology, GH; Resources, CL and PZ; Writing original draft, YS. All authors read and approved the final manuscript.

\section{Funding}

This research was funded by the National Natural Science Foundation of China [grant numbers 81430099 and 31500704 ], Projects of International Cooperation and Exchanges [grant number 2014DFA32950], research program from Beijing University of Chinese Medicine [grant number BUCM-2019-JCRC006 and 2019-JYB-TD013], CAMS Initiative for Innovative Medicine [grant number 2017-I2M-3-001], the Capital Medical Research and Development Fund [grant number 2016-4-40112], and the National Key Research and Development Program of China [grant number 2016YFC0901500]

\section{Availability of data and materials}

Original data of RNA-Seq has been submitted to www.ncbi.nlm.nih.gov/sra/. Data and materials are available from corresponding authors on reasonable request.

\section{Ethics approval and consent to participate}

This study was approved by the Institutional Review Board of Peking Union Medical College Hospital (protocol number: ZS-994). Consent to participate was acquired before recruitment from each participant.

\section{Consent for publication}

Not applicable.

\section{Competing interests}

The authors declare no conflict of interest. The funders had no role in the design of the study; in the collection, analyses, or interpretation of data; in the writing of the manuscript, or in the decision to publish the results.

\section{Author details}

${ }^{1}$ School of Life Sciences, Beijing University of Chinese Medicine, Beijing, China. ${ }^{2}$ Department of Traditional Chinese Medicine, Peking Union Medical College Hospital, Peking Union Medical College and Chinese Academy of Medical Sciences, Beijing, China. ${ }^{3}$ Department of Radiology, Peking Union Medical College Hospital, Peking Union Medical College and Chinese Academy of Medical Sciences, Beijing, China. ${ }^{4}$ Department of Nephrology, Peking Union Medical College Hospital, Peking Union Medical College and Chinese Academy of Medical Sciences, Beijing, China. ${ }^{5}$ State Key Laboratory of Biocontrol, Department of Biochemistry, School of Life Sciences, Sun Yat-Sen (Zhongshan) University, Guangzhou, Guangdong, China.

Received: 16 March 2019 Accepted: 26 July 2019

Published online: 08 August 2019

\section{References}

1. Chamot AM, et al. Acne-pustulosis-hyperostosis-osteitis syndrome. Results of a national survey. 85 cases. Rev Rhum Mal Osteoartic. 1987;54(3):187-96. 
2. Salles $M$, et al. The SAPHO syndrome: a clinical and imaging study. Clin Rheumatol. 2011;30(2):245-9.

3. Nguyen MT, et al. The SAPHO syndrome. Semin Arthritis Rheum. 2012;42(3):254-65.

4. Jurik $A G$, et al. SAPHO and CRMO: the value of imaging. Semin Musculoskelet Radiol. 2018:22(2):207-24.

5. Zimmermann P, Curtis N. Synovitis, acne, pustulosis, hyperostosis, and osteitis (SAPHO) syndrome - A challenging diagnosis not to be missed. J Infect. 2016;72 Suppl:S106-14.

6. Naves JE, et al. A systematic review of SAPHO syndrome and inflammatory bowel disease association. Dig Dis Sci. 2013;58(8):2138-47.

7. Marzano AV, et al. Pyoderma gangrenosum and its syndromic forms: evidence for a link with autoinflammation. Br J Dermatol. 2016;175(5):882-91.

8. Xu D, et al. Reduction of peripheral natural killer cells in patients with SAPHO syndrome. Clin Exp Rheumatol. 2019;37(1):12-8.

9. Firinu D, et al. TH17 cells are increased in the peripheral blood of patients with SAPHO syndrome. Autoimmunity. 2014;47(6):389-94

10. Liao HJ, et al. Increased neutrophil infiltration, IL-1 production and a SAPHO syndrome-like phenotype in PSTPIP2-deficient mice. Rheumatology (Oxford). 2015;54(7):1317-26.

11. Ferguson PJ, et al. Neutrophil dysfunction in a family with a SAPHO syndrome-like phenotype. Arthritis Rheum. 2008;58(10):3264-9.

12. Wekell $P$, et al. Neutrophils from patients with $\mathrm{SAPHO}$ syndrome show no signs of aberrant NADPH oxidase-dependent production of intracellular reactive oxygen species. Rheumatology (Oxford). 2016;55(8):1489-98.

13. Wang $X$, et al. Understanding the multifaceted role of neutrophils in Cancer and autoimmune diseases. Front Immunol. 2018:9:2456.

14. Tecchio C, Cassatella MA. Neutrophil-derived chemokines on the road to immunity. Semin Immunol. 2016;28(2):119-28.

15. $X u Z$, Wang $X$, Zheng $Y$. Screening for key genes and transcription factors in ankylosing spondylitis by RNA-Seq. Exp Ther Med. 2018;15(2):1394-402.

16. Aune TM, et al. Expression of long non-coding RNAs in autoimmunity and linkage to enhancer function and autoimmune disease risk genetic variants. J Autoimmun. 2017:81:99-109.

17. Kim D, Langmead B, Salzberg SL. HISAT: a fast spliced aligner with low memory requirements. Nat Methods. 2015;12(4):357-60.

18. Burgemeister LT, Baeten DL, Tas SW. Biologics for rare inflammatory diseases: TNF blockade in the SA PHO syndrome. Neth J Med. 2012;70(10):444-9.

19. Przepiera-Bedzak H, Fischer K, Brzosko M. Serum IL-6 and IL-23 levels and their correlation with Angiogenic cytokines and disease activity in ankylosing spondylitis, psoriatic arthritis, and SAPHO syndrome. Mediat Inflamm. 2015:2015:785705.

20. Zhang $S$, et al. Serum levels of proinflammatory, anti-inflammatory cytokines, and RANKL/OPG in synovitis, acne, pustulosis, hyperostosis, and osteitis (SAPHO) syndrome. Mod Rheumatol. 2019;29(3):523-30.

21. Przepiera-Bedzak H, Fischer K, Brzosko M. Serum Interleukin-18, Fetuin-a, soluble intercellular adhesion Molecule-1, and Endothelin-1 in ankylosing spondylitis, psoriatic arthritis, and SAPHO syndrome. Int J Mol Sci. 2016;17(8):e1255.

22. Sanna $\mathrm{M}$, et al. The salivary proteome profile in patients affected by SAPHO syndrome characterized by a top-down RP-HPLC-ESI-MS platform. Mol BioSyst. 2015:11(6):1552-62

23. Zhang $\mathrm{S}$, et al. Alteration of PTGS2 promoter methylation in chronic periodontitis. J Dent Res. 2010;89(2):133-7.

24. Geusens P, et al. NSAIDs and fracture healing. Curr Opin Rheumatol. 2013;25(4):524-31.

25. Chao CC, Hung FC, Chao JJ. Gas7 is required for mesenchymal stem cell-derived bone development. Stem Cells Int. 2013;2013:137010.

26. Bonaventura $\mathrm{P}$, et al. A feedback loop between inflammation and $\mathrm{Zn}$ uptake. PLoS One. 2016;11(2):e0147146.

27. Ogawa Y, et al. Zinc and skin disorders. Nutrients. 2018;10(2):e199.

28. Florea D, et al. Changes in zinc status and zinc transporters expression in whole blood of patients with systemic inflammatory response syndrome (SIRS). J Trace Elem Med Biol. 2018;49:202-9.

29. Aranda JF, et al. MYADM regulates Rac1 targeting to ordered membranes required for cell spreading and migration. Mol Biol Cell. 2011;22(8):1252-62.

30. Rahman A, Isenberg DA. Systemic lupus erythematosus. N Engl J Med. 2008;358(9):929-39.
31. Amoura $Z$, et al. Presence of antinucleosome autoantibodies in a restricted set of connective tissue diseases: antinucleosome antibodies of the lgG3 subclass are markers of renal pathogenicity in systemic lupus erythematosus. Arthritis Rheum. 2000;43(1):76-84.

32. Yeh $\mathrm{MH}$, et al. Extracellular matrix-receptor interaction signaling genes associated with inferior breast Cancer survival. Anticancer Res. 2018:38(8):4593-605.

33. Zhu N, et al. Identification of key genes in rheumatoid arthritis and osteoarthritis based on bioinformatics analysis. Medicine (Baltimore). 2018;97(22):e10997.

34. Flanagan $\mathrm{K}$, et al. Laminin-411 is a vascular ligand for MCAM and facilitates TH17 cell entry into the CNS. PLoS One. 2012;7(7):e40443.

35. Guezguez B, et al. Dual role of melanoma cell adhesion molecule (MCAM)/ CD146 in lymphocyte endothelium interaction: MCAM/CD146 promotes rolling via microvilli induction in lymphocyte and is an endothelial adhesion receptor. J Immunol. 2007;179(10):6673-85.

36. Kato $\mathrm{H}$, et al. Overproduction of Tenascin-C Driven by Lipid Accumulation in the Liver Aggravates Hepatic Ischemia/Reperfusion Injury in Steatotic Mice. Liver Transpl. 2019;25(2):288-301.

37. Maas SL, Soehnlein O, Viola JR. Organ-specific mechanisms of Transendothelial neutrophil migration in the lung, Liver, Kidney, and Aorta. Front Immunol. 2018:9:2739.

38. Miralda I, Uriarte SM, McLeish KR. Multiple phenotypic changes define neutrophil priming. Front Cell Infect Microbiol. 2017;7:217.

39. Moore $\mathrm{KL}$, et al. P-selectin glycoprotein ligand-1 mediates rolling of human neutrophils on P-selectin. J Cell Biol. 1995;128(4):661-71.

40. Yago T, et al. Selectins and chemokines use shared and distinct signals to activate beta2 integrins in neutrophils. Blood Adv. 2018;2(7):731-44.

41. Del Prete A, et al. The atypical receptor CCRL2 is required for CXCR2-dependent neutrophil recruitment and tissue damage. Blood. 2017;130(10):1223-34.

42. Kato $M$, et al. The novel endocytic and phagocytic C-type lectin receptor DCL-1/CD302 on macrophages is colocalized with F-actin, suggesting a role in cell adhesion and migration. J Immunol. 2007;179(9):6052-63.

43. Lozahic S, et al. CD46 (membrane cofactor protein) associates with multiple beta1 integrins and tetraspans. Eur J Immunol. 2000;30(3):900-7.

44. Walscheid K, et al. Elevated S100A8/A9 and S100A12 serum levels reflect intraocular inflammation in juvenile idiopathic arthritis-associated uveitis: results from a pilot study. Invest Ophthalmol Vis Sci. 2015;56(13):7653-60.

45. Tardif MR, et al. Secretion of S100A8, S100A9, and S100A12 by neutrophils involves reactive oxygen species and potassium efflux. J Immunol Res. 2015;2015:296149

46. Oury J, Oury F. Osteocalcin, a key molecule for bone endocrine functions. Med Sci (Paris). 2018:34(1):54-62.

47. Knight JS, Carmona-Rivera C, Kaplan MJ. Proteins derived from neutrophil extracellular traps may serve as self-antigens and mediate organ damage in autoimmune diseases. Front Immunol. 2012;3:380.

48. Delgado-Rizo $V$, et al. Neutrophil extracellular traps and its implications in inflammation: an overview. Front Immunol. 2017:8:81.

49. de Oliveira S, Rosowski EE, Huttenlocher A. Neutrophil migration in infection and wound repair: going forward in reverse. Nat Rev Immunol. 2016;16(6):378-91.

50. Langmead B, Salzberg SL. Fast gapped-read alignment with bowtie 2 . Nat Methods. 2012;9(4):357-9.

51. Roberts A, Pachter L. Streaming fragment assignment for real-time analysis of sequencing experiments. Nat Methods. 2013;10(1):71-3.

52. Love MI, Soneson C, Patro R. Swimming downstream: statistical analysis of differential transcript usage following Salmon quantification. F1000Res. 2018;7:952.

\section{Publisher's Note}

Springer Nature remains neutral with regard to jurisdictional claims in published maps and institutional affiliations. 